\title{
Melting of Moving Vortex Lattices in Systems With Periodic Pinning
}

\author{
C. Reichhardt and G.T. Zimányi \\ Department of Physics, University of California, Davis, Davis, CA 95616
}

(April 14, 2018)

\begin{abstract}
We study numerically the effects of temperature on moving vortex lattices interacting with periodic pinning arrays. For low temperatures the vortex lattice flows in channels, forming a hexatic structure with long range transverse and longitudinal ordering. At higher temperatures, a transition to a smectic state occurs where vortices wander between channels and longitudinal order is lost while transverse order remains. At the highest temperatures the vortex lattice melts into an isotropic liquid.

PACS numbers: 74.60.Ge, 74.60.Jg
\end{abstract}

Driven vortex lattices interacting with quenched and thermal disorder are an ideal system in which to study the nonequilibrium phases and transitions that arise from the interplay of competing interactions [1]7]. Transport measurements [5], neutron scattering [6], and Bitter decoration experiments [7] have provided strong evidence for transitions between different vortex dynamic phases, including creep, plastic flow, and ordered (elastic) flow. Theoretical work and simulations [1] suggested that for a moving vortex lattice, at high velocities the effect of disorder can be represented via a shaking temperature $T_{s h}$, inversely proportional to the velocity. At high velocities $T_{s h}$ decreases below the melting temperature $T_{m}$, and the vortices reorder. $T_{m}$ of the ordered moving vortex lattice is near the equilibrium $T_{m}$ of the disorder-free stationary lattice. In the highly driven, solidified state, theoretical [2] work suggested that the vortex lattice forms a moving Bragg-glass (MBG), or a strongly anisotropic moving smectic (MS) or moving transverse glass (MTG) [3]. In the MBG the vortices move in correlated channels with few defects, producing quasi-long range order. In the MS/MTG vortices move in uncorrelated channels, so although power law transverse order is present, the longitudinal order is short range only. Strongly anisotropic ordering, consistent with a MS/MTG, as well as more ordered phases, consistent with a MBG and vortex channeling, have been seen in simulations 佃 and Bitter decoration experiments [7].

Despite the considerable work done in the case of random quenched disorder, the effects of thermal disorder and melting for the interesting case of a moving vortex lattice interacting with a periodic pinning substrate have been far less studied. Periodic pinning substrates in superconductors can be created with arrays of microholes [8] and magnetic dots [9]. In all such systems, the pin radius is smaller than the distance between pins, so that a moving vortex spends the largest fraction of its time in the unpinned area. A crucial difference from the random pinning case is that the effect of the periodic pinning cannot be represented by a shaking temperature. The same periodicity also induces true long range transverse or longitudinal order in the moving lattice.
We report a numerical study of the melting of moving vortices in square periodic pinning arrays. With no driving, the system with pinning melts at a higher temperature than the pin free system. For moving vortices at low temperatures, we observe a triangular lattice flowing in strict $1 \mathrm{D}$ correlated channels, with transverse and longitudinal long range order. The transverse order is greater than the longitudinal order, and the anisotropy increases with temperature. At higher temperatures near the melting temperature of the clean equilibrium system, we observe a transition to a moving smectic (MS) state, where transverse vortex wandering between channels occurs. At even higher temperatures near the melting temperature of the pinned equilibrium system, the moving smectic melts into a moving isotropic liquid (ML). We present the dynamic phase diagram and explain its features in terms of the pinned and unpinned equilibium melting transitions.

We use finite temperature overdamped molecular dynamics simulations in two dimensions. $\mathbf{f}_{i}=\eta \mathbf{v}_{i}=$ $\mathbf{f}_{i}^{v v}+\mathbf{f}_{i}^{v p}+\mathbf{f}_{d}+\mathbf{f}_{i}^{T}$. We impose periodic boundary condition in $x$ and $y$. The force between vortices at $\mathbf{r}_{i}$ and $\mathbf{r}_{j}$ is $\mathbf{f}_{i}^{v v}=\sum_{j=1}^{N_{v}} f_{0} A_{v} K_{1}\left(\left|\mathbf{r}_{i}-\mathbf{r}_{j}\right| / \lambda\right) \hat{\mathbf{r}}_{i j}$, where $K_{1}(r / \lambda)$ is a modified Bessel function, $f_{0}=\Phi_{0}^{2} / 8 \pi^{2} \lambda^{3}, \lambda$ is the penetration depth and the parameter $A_{v}$ tunes the vortexvortex interaction strength. For most of the results here $A_{v}=1.0$. The driving force $\mathbf{f}_{d}$, representing a Lorentz force, is in the $x$ direction. The pinning is modeled as attractive parabolic traps of maximum strength $f_{p}$ and a range $r_{p}$ which is less than the distance between pins $a$. The thermal force $f_{i}^{T}$ has the properties $\left\langle f_{i}^{T}\right\rangle=0$ and $<f_{i}^{T}(t) f_{j}^{T}\left(t^{\prime}\right)>=2 \eta k_{B} T \delta_{i j} \delta\left(t-t^{\prime}\right)$. The temperature $T=\left(1 / 2 \eta k_{B}\right)\left(A f_{0}\right)^{2} \delta t$ where $\delta t$ is the time step in the simulation and $A$ is the number we tune to vary $T$, with $f_{i}^{T}=A f_{0}$. We take $f_{0}=k_{B}=\eta=1$ and use $\delta t=0.04$. We explore the phase diagram by conducting constant $f_{d}$ or $T$ sweeps on the $f_{d}-T$ plane. Our model is most relevant to superconductors with perodic arrays of columnar defects or thin-film superdonctors where the vortices can be approximated as $2 \mathrm{D}$ objects. A realistic $3 \mathrm{D}$ model would be needed to address the exact nature of the liquid phase, such as whether it is a line liquid. In 


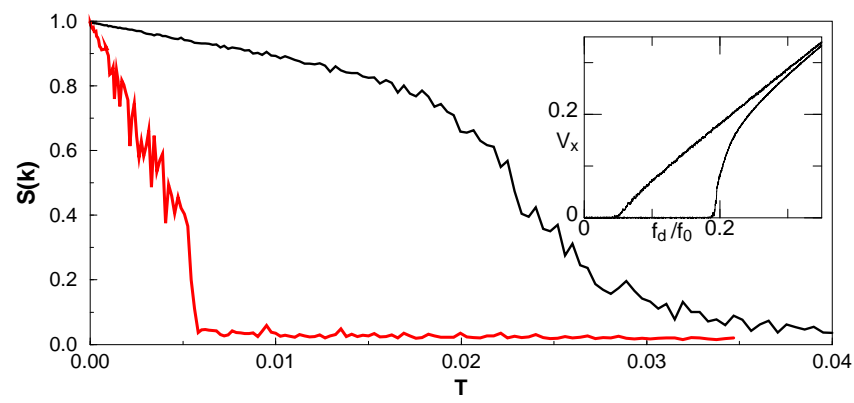

FIG. 1. The structure factor peaks $\langle S(k)\rangle / N_{v}$ versus $T$ for a system with $N_{v}=N_{p}$. Upper curve: Sample with a square pinning substrate of strength $f_{p} / f_{0}=0.22$. Lower curve: Sample containing no pinning. The melting for the pinned system occurs at much higher $T$ than melting in the clean system. Inset: $V_{x}$ versus $f_{d}$ with curves for $f_{p} / f_{0}=0.22$ and $T=0.0001$ and 0.0725 .

this work we consider only the commensurate case where the number of vortices $N_{v}$ equals the number of pinning sites $N_{p}$. Results for the incommensurate cases will be presented elsewhere. The initial vortex positions are generated by simulated annealing with each pinning site capturing one vortex. For finite size scaling we analyze system sizes with $N_{v} \sim L^{2}$ for $N_{v}=224$ to $N_{v}=2112$.

We first establish the melting temperature in the pin free and pinned systems without external drive $\left(f_{d}=0\right)$ with the vortex displacements $d_{r}=<|(\mathbf{r}(t)-\mathbf{r}(0))|^{2}>$, and the structure factor $S(\mathbf{k})=\frac{1}{L^{2}} \sum_{i, j} e^{i \mathbf{k} \cdot\left[\mathbf{r}_{i}(t)-\mathbf{r}_{j}(t)\right]}$. In the pinned system the vortex lattice has the same square symmetry as the pinning lattice. The melting temperature is determined from the simultaneous onset of diffusion and a drop in the peaks in $S(k)$. In Fig. 1 we show that the melting temperature $T_{m}$ is higher in the pinned system, with $T_{m}^{p} \approx 0.03$, than in the unpinned system, $T_{m}^{n p} \approx 0.0058$. This is reasonable since at commensuration the pins stiffen the vortex lattice.

Next we explore the dynamic phases of the system. For $f_{p}=0.22 f_{0}$ the $T=0$ depinning occurs at $f_{d}=0.22 f_{0}$. For fixed $f_{d}=0.45$ we perform a $T$ sweep, and monitor $S(\mathbf{k})$. Every $400 \mathrm{MD}$ steps we measure the transverse displacements $d_{y}=<|y(T)-y(0)|^{2}>$ from the initial positions of the vortices at $T=0$. For low drives the vortices form a pinned square vortex lattice. The pinned phase is defined by measuring $V_{x}=\left(1 / N_{v}\right) \sum_{i=1}^{N_{v}} \mathbf{v}_{i} \cdot \hat{\mathbf{x}}$. In the inset of Fig. 1 we show the typical $V_{x}$ versus $f_{d}$ curves for two different temperatures. The transition from the pinned to moving phases are marked by a jump in the $V_{x}$ at a well defined $f_{d}$. From $V_{x}$ versus $f_{d}$ curves we found little evidence for plastic or collective creep behaviour for temperatures below $T_{m}^{p}$; however, much longer time scales would be necessary to explore the creep behaviour.

Above some $T$ dependent driving force, we find that the vortices form a triangular lattice, with the principle lattice vector aligned with the direction of motion, since the vortices spend part of thier time between pins where



FIG. 2. (a) The transverse structure factor peak $S\left(k_{T}\right) / N_{v}$ (upper curve) and the longitudinal peak $S\left(k_{L}\right) / N_{v}$ (lower curve) for a $48 \lambda \times 48 \lambda$ system with $f_{d}=0.45 f_{0}$, $f_{p}=0.22 f_{p}$, and $N_{v}=N_{p}$. (b) The average transverse displacements of the vortices from their initial positions, $d_{y}=<|y(T)-y(0)|^{2}>$. Inset of (a): the scaling of $S(k) / N_{v}$ for different system sizes where $N_{v} \sim L^{2}$. For $\mathrm{T}=0.0025$ both the transverse (circles) and longitudinal peaks (squares and shifted down by 0.3 for clarity) scale as $S(k) \sim L^{0}$; for $\mathrm{T}=0.015$ the transverse peaks (up triangles) scale as $S\left(k_{T}\right) \sim L^{0}$ while the longitudinal peaks (left triangles) scale as $S\left(k_{L}\right) \sim L^{-1.96}$. Inset of (b): the evolution of the fraction of six-fold coordinated vortices $P_{6}$ versus temperature.

the vortex-vortex interaction dominates.

In Fig. 2(a) we plot the transverse $S\left(k_{T}\right)$ and longitudinal $S\left(k_{L}\right)$ structure function peaks for increasing $T$. For low $T, S\left(k_{T}\right)$ is only slightly larger than $S\left(k_{L}\right)$. This anisotropy between the peaks becomes more pronounced as $T$ is increased. The ordering can be analyzed from the finite size scaling of the structure factor as $S(k) / L^{2} \sim L^{-\eta}$ 11]. A solid with long range order will have $\eta=0$ while a system with short range order such as a liquid will have $\eta=2$. In the inset of Fig. 2(a), $S\left(k_{T}\right) / L^{2}$ and $S\left(k_{L}\right) / L^{2}$ (where $N_{v} \sim L^{2}$ ) are plotted for different system sizes in the low temperature regime ( $T=0.0025)$. Both peaks scale as $\eta \approx 0$, indicative of long range order. Near $T=0.0095$, which we label $T_{M S}$, $S\left(k_{L}\right)$ drops precipitously, indicating complete loss of longitudinal order, while $S\left(k_{T}\right)$ retains a significant finite value. Gradually $S\left(k_{T}\right)$ drops to the level of $S\left(k_{L}\right)$ near $T=0.03$, which we label $T_{M L}$. In this $T>T_{M S}$ regime the longitudinal peak $S\left(k_{L}\right) / L^{2}$ scales as $\eta=1.95 \pm 0.03$, consistent with an $\eta=2$ scaling behavior indicating the loss of longitudinal order. At this same temperature the transverse peak $S\left(k_{T}\right) / L^{2}$ shows a $\sim L^{-0.0}$ form (triangles in upper inset of Fig. 2), indicating that long range transverse order is still present. The behavior of the two peaks for $T_{M S}<T<T_{M L}$ indicates the presence of a moving smectic phase. For $T>T_{M L}$ both $S\left(k_{L}\right)$ and $S\left(k_{T}\right)$ scale as $\sim L^{-2}$ as the system becomes an isotropic liquid. We label these three phases the moving crystal 


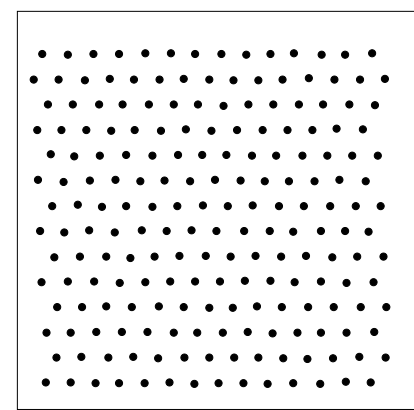

(a)

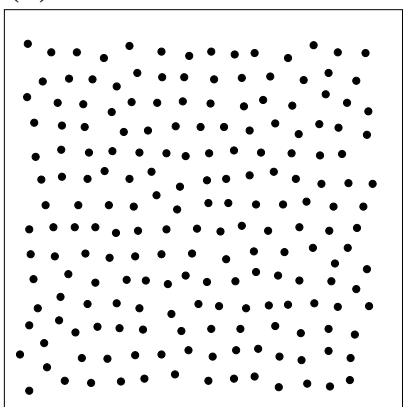

(c)

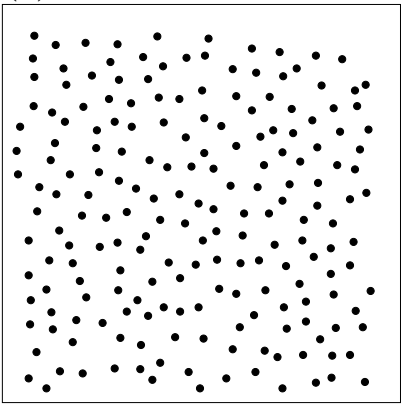

(e)

FIG. 3. The real space vortex images $(\mathrm{a}, \mathrm{c}, \mathrm{e})$ and vortex trajectories $(b, d, f)$ for the same system as in Fig. 2 for the moving lattice $(\mathrm{T}=0.0025)(\mathrm{a}, \mathrm{b})$; the moving smectic $(\mathrm{T}=$ $0.015)(\mathrm{c}, \mathrm{d})$; and the moving liquid $(\mathrm{T}=0.035)(\mathrm{e}, \mathrm{f})$.

'(MC), the moving smectic (MS) and moving liquid phase (ML). $\eta$ has some temperature dependence near the transitions which we will examine elsewhere. In the inset to Fig. 2(b) we plot the fraction of 6-fold coordination number $P_{6}$ versus $T$ as obtained from the Voronoi construction. The proliferation of defects occurs at $T=T_{M S}$ as seen by the drop in $P_{6}$. In Fig.2(b) we plot the transverse displacement $d_{y}$. For $T<T_{M S}, d_{y} \approx 0$ indicating that the vortices are moving in straight $1 \mathrm{D}$ channels. For $T \geq T_{M S}, d_{y}$ increases indicating that the onset of vortex wondering in the $y$ direction is correlated with the loss of longitudinal order and the proliferation of defects.

To examine the individual vortex behavior in the different dynamic phases in Fig. 3 we plot snapshots of the vortex positions and trajectories for $\mathrm{MC}$ at $T=0.0025$, MS at $T=0.015$, and ML at $T=0.035$. In the MC phase, the vortices form an ordered triangular lattice [Fig.3(a)], and move in correlated 1D paths [Fig.3(b)], which agrees

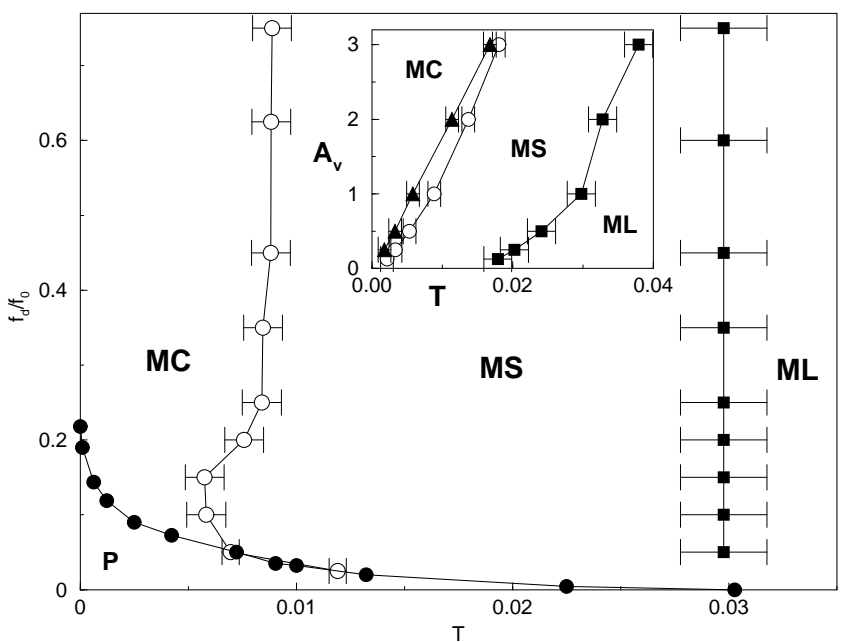

FIG. 4. The dynamic phase diagram of $f_{d}$ versus $T$. For open circles and filled squares, the current is fixed and the temperature is increased. For the filled circles, the temperature is fixed and the drive is increased. The pinned to moving phases are determined from $f_{d}$ versus vortex velocity $V_{x}$. The boundaries of the other phases are determined from transverse and longitudinal $\langle S(k)\rangle$ and $d_{y}$. The open circles correspond to the MC to ML transition and the filled squares correspond to the MS to ML transition for a system with the same parameters in Fig. 2. The filled circles correspond to the transition out of the pinned phase P. Inset: The phase diagram for $A_{v}$, the vortex-vortex interaction prefactor, versus $T$. The triangles correspond to the zero drive clean melting temperature while the open circles and squares correspond to the MC-ML transition and the MS-ML transition with $f_{d}=0.45 f_{0}$.

with the zero transverse wandering $d_{y} \approx 0$ in Fig. 2(b). As $T$ is increased in the MC phase the channel width increases; however, vortices do not cross from one channel to another. The pinning also induces the long-range order seen from the scaling of $S(k)$, whereas algebraic decay would be expected since the system is in $2 \mathrm{D}$.

In the MS phase, shown in Fig. 3(c), the vortices are much less ordered than in the MC phase; however, some order remains. The vortex trajectories in the MS phase, Fig. 3(d), reveal that although some channeling occurs along the pinning, there is considerable vortex motion between and across the channels. This interchannel vortex motion accounts for the increase of the transverse displacements, $d_{y}$, at the onset of the MS phase in Fig. 2(b). The residual vortex channeling accounts for the finite value of $S\left(k_{T}\right)$ in the MS phase, but the vortex positions are uncorrelated between channels so there is no longitudinal order and the vortex lattice is highly defected. Since the residual channels have the same period as the pinning lattice the transverse scaling in $S(k)$ gives long range order. Finally in the ML phase, shown in Fig.3(e,f), the vortex positions are disordered and the channeling behavior is lost.

In Fig. 4 we present the central result of this work, 
the dynamic phase diagram as a function of $f_{d}$ and $T$ obtained from fixed drive increasing $T$ simulations (denoted by solid circles) and from fixed temperature increasing drive simulations (denoted by open circles and squares). The depinning line is determined from the pronounced upward curvature in the $V(I)$ curves or the onset of displacements. The MC-MS line is found from the saturation of $S\left(k_{L}\right)$ to a minimum value as well as the onset of the transverse displacements. The MS-ML line is obtained from the point at which the transverse $S\left(k_{T}\right)$ peak drops to a minimal value and exhibits a scaling of $\eta \approx 2$. The MS-ML line is roughly independent of drive above $f_{d}=0.25 f_{0}$. The MS line shows some curvature toward lower temperatures for drives near the depinning line. The MC-MS transition occurs a small amount above $T_{m}^{n p}=0.0058$ but approaches this value for the lower drives. The MS-ML transition line coincides with the pinned (zero driving value) melting temperature $T_{m}^{p}$. The pinned phase vanishes at $T_{m}^{p}$.

The onset of these different phases can be understood by considering that for higher drives the moving vortices spend a great part of their time outside the pinning sites because $r_{p} \lesssim a$. For temperatures above $T_{m}^{n p}$ the vortices enter a molten state while moving between pinning sites since they are essentially moving in a clean system. In this melted state, the thermal fluctuations overcome the vortex-vortex interaction and the correlation of vortices in adjacent channels as well as the longitudinal order is lost. The vortices start diffusing at random, leading to a large increase in $d_{y}$. Unlike the case of random pinning, which induces an additional shaking temperature that effectively lowers the temperature at which the vortices disorder or melt, vortices moving in a periodic pinning array will not experience a shaking temperature. As long as $T$ is less than the melting temperature of the nondriven clean system $T_{m}^{n p}$, at any drive the overall moving vortex lattice remains ordered. Further there is still a pinning effect in the transverse direction. This transverse pinning which causes the channeling has been seen in simulations with random pinning [4] and is particularly large for simulations with periodic pinning arrays 10 . During the time the vortices are in the pinning sites they still feel a transverse pinning force until $T>T_{m}^{p}$, so some vortex channeling persists and finite transverse ordering appears. Above $T_{m}^{p}$ the pinning is no longer effective so channeling and transverse order are both lost.

A test of the above interpretation is that $T_{M S}$ is related to $T_{m}^{n p}$. $T_{m}^{n p}$ can be tuned by changing the vortex-vortex interaction prefactor $A_{v}$. As $A_{v}$ is lowered $T_{m}^{n p}$ will also be lowered. In the inset of Fig. 4 we show the melting lines for the clean non-moving system and the moving system with pinning $\left(f_{d}=0.45 f_{0}\right)$ for $T$ versus $A_{v}$. As $A_{v}$ is increased the $T_{m}^{n p}$ and $T_{M S}$ lines increase at the same rate. This correlation is encouraging evidence for the above interpretation. For the stiffer lattices (high $A_{v}$ ) the MC phase is larger.
In summary, we have studied numerically the melting and dynamic phase diagram of a vortex lattice interacting with a square periodic pinning array. The melting temperature for the non-driven pinned system is higher than that for the equivalent clean system. For moving vortices at low $T$ the vortex lattice moves in correlated $1 \mathrm{D}$ channels and has long range order with some anisotropy between the longitudinal and transverse peaks. At a higher temperature there is a transition to a Moving Smectic phase where longitudinal order is lost while long-range transverse order remains. In the Moving Smectic phase the channel flow still persists but vortices diffuse between channels. At high temperatures the transverse order and channeling is also lost. The Moving Crystal-Moving Smectic transition corresponds roughly to the melting temperature of the zero drive clean system.

We thank C.J. Olson and R.T. Scalettar for useful discussions and critical reading of the manuscript. Funding provided by CLC/CULAR.

- Note Added After submission we became aware of the paper by V. Marconi and D. Dominguez [12] in which they also study the melting of moving vortex lattices intercting with a periodic substrate in a peroidc Josephsonjuntion array in which we find several overlapping results. They, however, do not find a moving smectic phase which is due to their pinning being modeled as an egg-carton potential unlike our model in which the space between the pinned sites is essential for the smectic phase to occur.

[1] A.E. Koshelev and V.M. Vinokur, Phys. Rev. Lett. 73, 3580 (1994).

[2] T. Giamarchi and P. Le Doussal, Phys. Rev. Lett. 76, 3408 (1996).

[3] T. Giamarchi and P. Le Doussal, Phys. Rev. B 57, 11 356 (1998); L. Balents, M.C. Marchetti, and L. Radzihovsky, ibid. 57, 7705 (1998); S. Scheidl and V.M. Vinokur, Phys. Rev. E 57, 2574 (1998).

[4] K. Moon, R.T. Scalettar, and G.T. Zimányi, Phys. Rev. Lett. 77, 2778 (1996); S. Ryu et al., ibid. 77, 5114 (1996); S. Spencer and H.J. Jensen, Phys. Rev. B 55, 8473 (1997). D. Dominguez et al., Phys. Rev. Lett. 78, 2644 (1997); C. J. Olson, C. Reichhardt, and F. Nori, ibid. 81, 3757 (1998). D. Dominguez, ibid. 82, 181 (1999). A. Kolton et al., ibid. 83, 3061 (1999).

[5] S. Bhattacharya and M.J. Higgins, Phys. Rev. Lett. 70, 2617 (1993); M.C. Hellerqvist et al., ibid. 76, 4022 (1996); W. Henderson et al., ibid. 77, 2077 (1996).

[6] U. Yaron et al., Nature 376, 753 (1995).

[7] F. Pardo et al., Phys. Rev. Lett. 78, 2644 (1997). M. Marchevsky et al., Phys. Rev. Lett. 78, 531 (1997). F. Pardo et al., Nature 396, 348 (1998).

[8] M. Baert et al., Phys. Rev. Lett. 74, 3269 (1995); K. Harada et al., Science 271, 1393 (1996); A. Castellanos et al., Appl. Phys. Lett. 71, 962 (1997); V. Metlushko et al., Phys. Rev. B 59, 603 (1999). L. Van Look et al., ibid, 60, R6998 (1999).

[9] J.I. Martín et al., Phys. Rev. Lett. 79, 1929 (1997); 
83, 1022 (1999); D.J. Morgan and J.B. Ketterson, Phys. Rev. Lett. 80, 3614 (1998); Y. Fasano et al., to be published.

[10] C. Reichhardt, C.J. Olson and F. Nori, Phys. Rev. Lett. 78, 2648 (1997); C. Reichhardt, C.J. Olson and F. Nori, Phys. Rev. B 58, 6534 (1998).

[11] M. Franz and S. Teitel, Phys. Rev. B 51, 6551 (1995).

[12] V. Marconi and D. Dominguez, Phys. Rev. Lett. 83, 3061 (1999). 